\title{
Álcool e alcoolismo: atitudes de estudantes de Enfermagem
}

\author{
Alcohol and alcoholism: attitudes of nursing students \\ Alcohol y alcoholismo: actitudes de estudiantes de Enfermería
}

\begin{abstract}
Divane Vargas', Marina Nolli Bittencourt"
' Universidade de São Paulo, Escola de Enfermagem, Departamento de Enfermagem Materno-Infantil e Psiquiátrica, Programa de Pós-Graduação em Enfermagem. São Paulo-SP, Brasil.

"Universidade de São Paulo, Escola de Enfermagem, Departamento de Enfermagem Materno-Infantil e Psiquiátrica, Programa de Pós-Graduação em Enfermagem (Doutoranda). São Paulo-SP, Brasil.
\end{abstract}

Submissão: 12-05-2011

Aprovação: 08-02-2013

\section{RESUMO}

Estudo descritivo exploratório que objetivou verificar as atitudes dos estudantes de enfermagem frente à bebida alcoólica, ao alcoolismo e ao alcoolista segundo sua posição frente aos itens de uma escala de atitudes. Para coleta dos dados, utilizou-se a Escala de Atitudes Frente ao Álcool, ao Alcoolismo e ao Alcoolista, aplicada a 144 estudantes de enfermagem. Evidenciouse tendência a atitudes negativas desses estudantes frente ao alcoolismo, ao alcoolista e à bebida alcoólica, pois a maioria se colocou na categoria indiferente ou discordo dos itens positivos, concordando com os itens negativos da escala. Conclui-se que essa tendência a atitudes negativas deve-se à pouca atenção dada à temática durante a formação do enfermeiro, constatando-se a necessidade de que maior importância seja dada a essa problemática.

Descritores: Atitude; Alcoolismo; Estudantes de Enfermagem.

\section{ABSTRACT}

This is a descriptive exploratory study that aimed to verify nursing students' attitudes facing to the alcoholic drinks, alcoholism and alcoholics, according to their position in face of an attitudes scale items. For data collection, it was used the Scale of Attitudes to alcohol, alcoholism and alcoholic, applied to 144 nursing students. The results showed a tendency to negative attitudes of these students in face of alcoholism, alcoholic person and alcoholic drinks, since most participants were placed in category indifferent or disagree with the positive items, agreeing with negative scale items. We conclude that this trend of negative attitudes is connected to insufficient attention given to the subject during the nurses' education, being verified the need for greater importance to be given to this problem.

Key words: Attitude; Alcoholism; Nursing Students.

\section{RESUMEN}

Estudio exploratorio descriptivo, que tuvo como objetivo conocer las actitudes de los estudiantes de enfermería frente a la bebida alcohólica, el alcoholismo, y al alcohólicos, de acuerdo a su posición ante los ítems de una escala de actitudes. Para la recolección de datos, se utilizo la Escala de Actitudes frente al alcohol, al alcoholismo y al alcohólico, aplicada a 144 estudiantes de enfermería. Los resultados muestran una tendencia a actitudes negativas de estos estudiantes frente al alcoholismo, las bebidas alcohólicas y a los alcohólicos, desde que la mayoría de los participantes fueron clasificados en la categoría indiferentes o no de acuerdo con los elementos positivos, y de acuerdo con los elementos negativos de la escala. Llega-se a la conclusión de que esta tendencia de actitudes negativas es debida a la insuficiente atención al tema durante la formación de los enfermeros, observando-se la necesidad de que sea dada mayor importancia a este problema.

Palabras clave: Actitud; Alcoholismo; Estudiantes de Enfermería. 


\section{INTRODUÇÃO}

De acordo com o relatório sobre a saúde global (2002), o álcool foi responsável por $4 \%$ das taxas de mortalidade e 3,2\% (1,8 milhões) das mortes globais em 2000 ${ }^{(1)}$. A Organização Mundial de Saúde considerou o álcool como o $5^{\circ}$ mais importante fator de risco para mortes prematuras ${ }^{(1)}$. Esses dados se tornam ainda mais preocupantes quando se constata que, cada vez mais, rejuvenesce a faixa etária que faz uso dessa substância, pois, o beber de forma compulsiva, especialmente em jovens, tem aumentado em muitos países da região das Américas, incluindo México, Brasil, Peru, Uruguai e Chile ${ }^{(2)}$. Nesse continente, o consumo de álcool é aproximadamente $40 \%$ maior que a média mundial, o que pode justificar o fato de que o continente americano é a única região do mundo onde o álcool está como primeiro causador de mortes prematuras e incapacidades ${ }^{(1)}$.

No Brasil, as repercussões do consumo abusivo de álcool são evidentes, isso pode ser observado por meio dos resultados do I e II Levantamento Domiciliar Nacional ${ }^{(3-4)}$ sobre o uso de drogas psicotrópicas realizados nos anos 2001 e 2005, respectivamente, envolvendo as maiores cidades do Brasil. Conforme o I Levantamento, $68,7 \%$ das pessoas relataram ter feito o uso do álcool uma vez na vida, passando para $74,6 \%$ no II Levantamento; ainda de acordo com os dados dos dois levantamentos, a estimativa de dependentes de bebidas alcoólicas no país passou de 11,2\% em 2001 para 12,3\% em 2005, sendo que a maior proporção de dependentes de álcool está entre os homens. Apesar da constatação de que, atualmente, os problemas relacionados ao álcool constituem um dos maiores problemas de saúde pública brasileira ${ }^{(5)}$, quando se compara a frequência de pessoas que receberam tratamento devido ao uso de álcool e outras drogas entre 2001 e 2005, houve uma diminuição de $4,0 \%$, em 2001, para $2,9 \%$, em $2005^{(4)}$.

Ainda assim, são elevadas as taxas de pacientes com problemas relacionados ao álcool e alcoolismo ocupando leitos de unidades clínicas, cirúrgicas ou de emergência de hospitais gerais do país( ${ }^{(6)}$, bem como nos dispositivos de atenção primária a saúde ${ }^{(7)}$. Diante dessas constatações é provável que o maior contato do paciente com problemas decorrentes do uso de álcool em todos esses serviços seja com o enfermeiro e sua equipe. $E$, pensando que os estudantes de enfermagem passam por esses serviços durante seus estágios na graduação, eles também terão maior contato com esse paciente.

Especialistas têm evidenciado que cuidar de pacientes com problemas relacionados ao álcool e outras drogas nem sempre constitui tarefa fácil para o enfermeiro generalista ${ }^{(8)}$, sendo comuns atitudes negativas frente a essa clientela, haja vista o pouco preparo e falta de conhecimentos e habilidades adequadas para prestar cuidado ${ }^{(9)}$. Considerando as evidências da literatura que apontam ${ }^{(10)}$ a carência de conhecimentos em relação ao álcool, alcoolismo e ao alcoolista, além do despreparo dos enfermeiros no cuidado ao dependente químico, demonstrado por suas atitudes negativas, compreende-se que os estudantes de enfermagem também demonstrem tal comportamento durante sua prática hospitalar, uma vez que é durante a formação acadêmica que se adquire os conhecimentos necessários para a formação também das atitudes, sendo elas positivas ou não, em relação ao alcoolista. Por isso a importância de se conhecer as atitudes dos estudantes de enfermagem frente a essa clientela.

A revisão da literatura sobre as atitudes dos estudantes de enfermagem frente ao álcool, ao alcoolismo e ao alcoolista evidencia que pouco tem se investigado sobre essa temática no Brasil. Além disso, tem evidenciado que os estudos disponíveis não se referem exclusivamente às atitudes dos estudantes de enfermagem $^{(11)}$. Na literatura internacional, sobretudo na norte-americana, é possível encontrar algumas publicações, surgidas nas últimas décadas ${ }^{(11)}$, relacionadas à temática em questão.

Estudos realizados nos Estados Unidos ${ }^{(12-13)}$ constataram que os estudantes de enfermagem apresentavam atitudes negativas e pouco aceitáveis a respeito do alcoolista e do alcoolismo. No Brasil, dos poucos estudos relacionados a atitudes dos estudantes de enfermagem com questões como o álcool, o alcoolismo e o alcoolista, evidenciaram que os estudantes consideram o alcoolista uma pessoa sem caráter e personalidade fraca ${ }^{(14)} \mathrm{e}$ ainda revelam que o alcoolismo constitui-se numa das patologias mais rejeitadas pelos alunos de enfermagem ${ }^{(15)}$.

Pesquisa realizada com estudantes de enfermagem e docentes, com o objetivo de conhecer as representações sociais que esses sujeitos tinham do indivíduo alcoolista, demonstrou que sua realidade representacional não diferia da representação do senso comum, pois carregava as discriminações e os estereótipos da sociedade onde estavam inseridos ${ }^{(16)}$.

Outro estudo, que teve como objetivo avaliar os conteúdos teórico-práticos sobre o uso do álcool e suas consequências, adquiridos por enfermeiros, estudantes e docentes de enfermagem durante a formação na graduação e pós-graduação, além de avaliar a relação desses conteúdos e a percepção desses sujeitos frente à questão, demonstrou, dentre as conclusões, que os estudantes de enfermagem percebiam menos as intervenções utilizadas nos cuidados de enfermagem aos alcoolistas do que os enfermeiros, e que os docentes percebiam o alcoolismo mais como uma questão de privacidade do que os enfermeiros e os estudantes de enfermagem ${ }^{(17)}$.

Considerando os dados epidemiológicos, que demonstram a prevalência dos problemas relacionados ao álcool e ao alcoolismo na população brasileira, o fato dos estudos disponíveis no Brasil não se ocuparem em estudar especificamente as atitudes da população de estudantes de enfermagem em relação ao álcool, alcoolismo e ao alcoolista; e considerando que as atitudes e habilidades dos estudantes de enfermagem constituem elementos capazes de interferir no cuidado a essa clientela, esse estudo é relevante uma vez que, ao identificar as atitudes adquiridas durante a graduação do enfermeiro, é possível que ainda se possa intervir em sua formação, para minimizar as atitudes negativas que possam ter aparecido. Por isso, este estudo objetiva verificar as atitudes de estudantes de enfermagem frente à bebida alcoólica, ao alcoolismo e ao alcoolista, segundo sua posição frente aos itens de uma escala de atitudes.

\section{METODOLOGIA}

Tratou-se de um estudo descritivo exploratório em que, para coleta dos dados, se aplicou a Escala de Atitudes Frente ao Álcool, 
ao Alcoolismo e ao Alcoolista (EAFAAA) numa amostra composta por 144 estudantes do último ano de graduação em enfermagem, que concordaram em participar do estudo, provenientes de duas escolas de enfermagem de uma cidade do interior de São Paulo. Essas escolas foram escolhidas como cenário da pesquisa por tratarem-se de locais onde um dos autores realizava atividades de ensino no momento da coleta.

Ressalta-se que a EAFAAA é constituída por 96 itens distribuídos em cinco fatores (F1: O alcoolista: o trabalho e as relações interpessoais; F2: Etiologia; F3: Doença; F4: As repercussões decorrentes do uso/abuso do álcool; F5: Bebidas alcoólicas). Foi validada em 2005, e apresentou um índice de confiabilidade de $0,90^{(8)}$. Uma das características desse instrumento é a predominância de itens negativos, pois, dos 96 itens que o compõe, 72 são itens negativos (75\%), ou seja, são itens que revelam atitudes negativas, ou contrárias, ao alcoolista, ao alcoolismo e ao álcool. Segundo o autor da Escala, ao apresentar-se predominantemente com itens negativos, quanto maior o desacordo do sujeito em relação ao item, mais positiva é a sua atitude.

Apesar de a EAFAAA ser constituída por 5 fatores, nesse estudo optou-se somente pela análise e apresentação de três deles (1- O alcoolista: o trabalho e as relações interpessoais; 2- Etiologia do alcoolismo; 5- bebida alcoólica), uma vez que estes são os fatores que avaliam, especificamente, as atitudes dos estudantes frente ao álcool, ao alcoolismo e ao alcoolista, conforme o objetivo do presente estudo. Esses três fatores, assim como os demais fatores da EAFAAA, apresentam predominância de itens negativos (Gráfico 1).

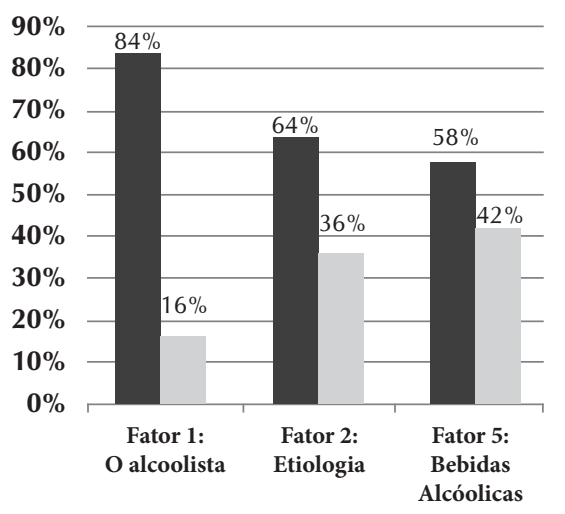

itens negativos itens positivos

Gráfico 1 - Frequência de itens positivos e negativos nos fatores 1, 2 e 5 da EAFAAA. São Paulo, 2011.

Quanto à descrição operacional dos três fatores investigados nesse estudo, verifica-se que o Fator 1 refere-se à percepção, às opiniões e aos sentimentos frente as características comportamentais e psíquicas do indivíduo alcoolista, bem como o trabalhar e o relacionar-se com ele; o Fator 2 (A etiologia do alcoolismo) traz concepções, opiniões e atitudes sobre a etiologia do alcoolismo, fatores psíquicos morais e biológicos que podem ser atribuídos como causa do alcoolismo; e o Fator 5 (Bebida alcoólica) é composto por itens que envolvem opiniões, sentimentos e condutas frente a bebida alcoólica; consequências trazidas pelo uso das bebidas ao individuo; o limite entre o beber e o normal e o patológico; efeitos da bebida sobre o comportamento da pessoa.
Cada um dos itens que compunham o instrumento entregue aos sujeitos podia ser respondido por meio de uma escala do tipo likert de cinco pontos: ( $1=$ Discordo totalmente; $2=$ Discordo; 3 =Indiferente; 4 =Concordo; 5 =Concordo totalmente). Para fins de apresentação neste estudo, agruparam-se os itens 1- discordo totalmente e 2- discordo em 1-discordo, 2não concordo nem discordo (neutralidade) e 4- concordo e 5- concordo totalmente em 3- concordo. Juntamente com a EAFAAA aplicou-se um questionário sociodemográfico que continha os dados pessoais dos sujeitos do estudo (Escola, sexo e idade). Os instrumentos de coleta foram entregues aos participantes em envelopes lacrados. Solicitou-se que os questionários não fossem identificados, a fim de se garantir a privacidade dos sujeitos. Os participantes levaram aproximadamente 30 minutos para responder os instrumentos.

\section{Análise dos dados}

De posse dos instrumentos respondidos $(N=144)$, criou-se um banco de dados no programa Statistical Package for the Social Sciences v.13 for Windows (SPSS) ${ }^{\circledR}$, através do qual se procedeu à análise estatística dos dados. Essa análise foi realizada por meio de estatísticas descritivas e utilizaram-se tabelas de frequência, valores de média e mediana, desvio padrão, variância e intervalo de confiança para apresentação dos dados.

\section{Aspectos éticos}

Os procedimentos éticos desse estudo estão representados pela aprovação do projeto de estudo por parte do Comitê de Ética em Pesquisa com seres humanos da Escola de Enfermagem da Universidade de São Paulo, Parecer n 710/2008, e pela assinatura, por parte dos sujeitos participantes da pesquisa, do Termo de Consentimento Livre e Esclarecido (TCLE).

\section{RESULTADOS}

\section{Perfil da amostra}

Segundo os dados da tabela 1, observa-se que a maioria dos participantes era do sexo feminino (73\%) e da escola B $(62 \%)$. Em relação à idade, observa-se que a maior proporção dos estudantes de enfermagem estava na faixa etária de 19 a 25 anos de idade (43\%).

Tabela 1 - Frequência dos dados pessoais dos estudantes de Enfermagem, Ribeirão Preto-SP, 2010.

\begin{tabular}{ccc}
\hline \multirow{2}{*}{ Variáveis } & \multicolumn{2}{c}{ Estudantes } \\
\cline { 2 - 3 } & $\mathbf{N}$ & $\%$ \\
\hline Sexo & 122 & \\
Feminino & 22 & 85 \\
Masculino & $\mathbf{1 4 4}$ & 15 \\
Total & & $\mathbf{1 0 0}$ \\
Idade & 84 & \\
$19-25$ & 29 & 59 \\
$26-30$ & 11 & 20 \\
$31-35$ & & 8 \\
\hline
\end{tabular}




\begin{tabular}{ccc}
\hline \multirow{2}{*}{ Variáveis } & \multicolumn{2}{c}{ Estudantes } \\
\cline { 2 - 3 } & $\mathrm{N}$ & $\%$ \\
\hline Idade & & \\
$36-40$ & 10 & 7 \\
41 ou + & 9 & $\mathbf{1 0 0}$ \\
Total & $\mathbf{1 4 4}$ & \\
Local de Estudo & & 38 \\
Escola A & 55 & 62 \\
Escola B & 89 & $\mathbf{1 0 0}$ \\
Total & $\mathbf{1 4 4}$ &
\end{tabular}

\section{Análise das respostas aos itens}

De acordo com os dados do Gráfico 2, relacionado ao fator 1 (O alcoolista) da escala de atitudes, observou-se que a maior parte $(38 \%)$ dos participantes colocou-se em categoria indiferente aos itens positivos da escala, seguidos de $37 \%$ dos que discordam com os itens negativos e $35 \%$ daqueles que se consideram indiferentes aos itens negativos da escala.

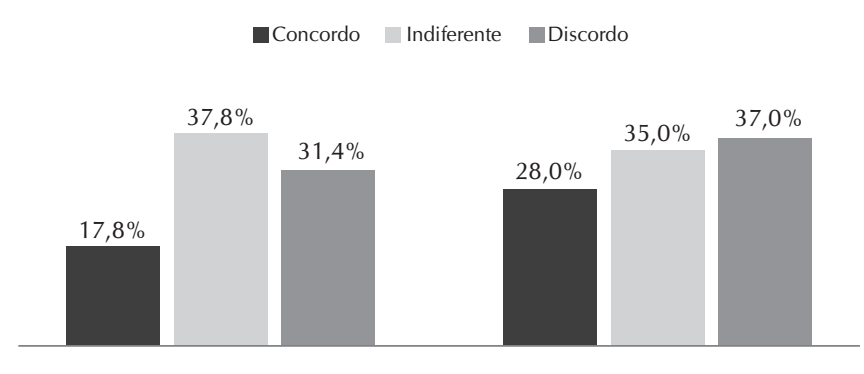

Itens Positivos

Itens Negativos

Gráfico 2 - Distribuição dos sujeitos de acordo com as respostas aos itens positivos e negativos do Fator 1: $\mathrm{O}$ alcoolista. São Paulo, 2010.

Conforme os dados do Gráfico 3, relacionado ao fator 2 (Etiologia do alcoolismo) da escala de atitudes, observou que a maioria (58\%) dos participantes colocou-se em categoria indiferente aos itens positivos da escala, seguidos de $43 \%$ dos que concordam com itens negativos e $32 \%$ dos que discordam com os itens positivos.
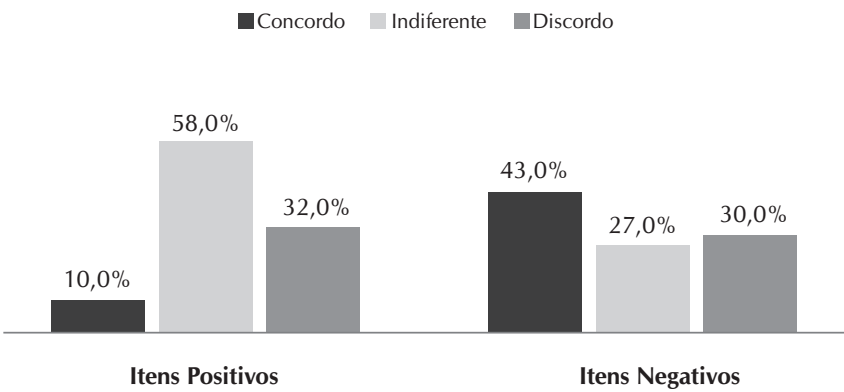

Gráfico 3 - Distribuição dos sujeitos de acordo com as respostas aos itens positivos e negativos do Fator 2: Etiologia do alcoolismo. São Paulo, 2010.
De acordo com os dados do Gráfico 4, relacionado ao fator 5 (Bebidas alcoólicas) da escala de atitudes, observou-se que a maior parte (48\%) dos participantes colocou-se em categoria indiferente aos itens positivos da escala, seguidos de $42 \%$ dos que discordaram com itens positivos e $32 \%$ daqueles que concordaram com os itens negativos.

—Concordo Indiferente Discordo

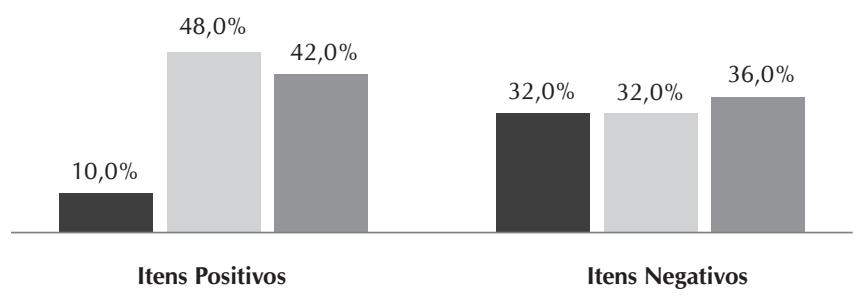

Gráfico 4 - Distribuição dos sujeitos de acordo com as respostas aos itens positivos e negativos do Fator 5: Bebidas alcoólicas. São Paulo, 2010

\section{DISCUSSÃO}

Com relação às atitudes dos estudantes de enfermagem frente aos três fatores analisados, houve uma tendência a atitudes negativas, uma vez que grande parte não apresentou atitude formada com relação aos fatores. Essa afirmação se comprova, pelo fato de muitos dos respondentes terem se alocado na categoria indiferente, frente aos itens positivos da subescala; aliado a isso, tenderam a discordar dos itens positivos.

De acordo com estudo anterior ${ }^{(8)}$, indivíduos com alta pontuação tendem a apresentar atitudes positivas enquanto indivíduos com baixa pontuação tendem a apresentar atitudes negativas, ou seja, aqueles que tendem a concordar com itens negativos tendem a apresentar atitudes negativas. A constatação de atitudes negativas por parte dos estudantes de enfermagem encontrada nesse estudo são consistentes, se comparada aos resultados de pesquisas ${ }^{(11-12,17)}$ semelhantes e que evidenciaram o predomínio de atitudes negativas nessa população.

A alta frequência de respostas indiferente, tanto aos itens negativos quanto aos itens positivos dos fatores analisados, parece demonstrar que os estudantes de enfermagem não se sentem confortáveis e nem aptos a se posicionarem frente aos itens da escala, fato que pode ser explicado como consequência da falta de conhecimento desses estudantes em relação à temática álcool. Outro fator que pode explicar essa situação é a dificuldade de assumirem suas reais atitudes, como se fosse mais confortável então para os mesmos colocarem-se em posições neutras ou sem uma opinião formada.

O resultado acima apresentado é corroborado pelos resultados de um estudo que avaliou o conhecimento dos estudantes de enfermagem na temática álcool e outras drogas, constatando que o grande percentual de alunos que não responderam as questões ou que manifestaram desconhecimento sobre os itens do questionário aplicado poderia estar relacionado à pouca carga horária destinada a esse conteúdo durante a graduação ${ }^{(17)}$.

No que se refere às atitudes dos estudantes de enfermagem frente ao Fator 1 (O alcoolista: o trabalho e as relações 
interpessoais), a alta frequência de resposta indiferente aos itens positivos e negativos e a tendência em concordar com os itens negativos, demonstra uma inclinação a atitudes negativas por parte desses estudantes e que concernem às características comportamentais e psíquicas do indivíduo alcoolista, bem como o trabalhar e o relacionar-se com essa população.

A esse respeito, embora se analisando o comportamento de enfermeiros, um estudo ${ }^{(18)}$ constatou que os mesmos não se sentiam satisfeitos em trabalhar com pacientes alcoolistas. Esse resultado se apresenta preocupante, uma vez que pesquisa aponta que as características atribuídas ao alcoolista vão influenciar diretamente na sua atuação com esse indivíduo, levando o aluno a aceitar ou a rejeitar esse paciente ${ }^{(14)}$. Além disso, uma vez que os estudantes dessa pesquisa demonstraram atitudes negativas em relação às características comportamentais e psíquicas do alcoolista, eles podem rejeitar esse paciente, pois, conforme o mesmo autor, as características comportamentais dos pacientes são o principal responsável pela rejeição do alcoolista, sendo apontada por $43,4 \%$ dos estudantes do seu estudo.

No que se refere ao fator 2 (a etiologia do alcoolismo), a alta frequência da resposta indiferente aos itens positivos e a maior concordância aos itens negativos, também demonstra uma tendência a atitudes negativas por parte dos estudantes de enfermagem em relação à etiologia do alcoolismo. Fatores psíquicos, morais e biológicos que podem são atribuídos como causas do alcoolismo.

Tal fato pode estar relacionado à carência de preparo educacional durante a graduação em relação à temática do álcool, ou a falta de abordagem apropriada do conteúdo nas universidades, devido à deficiência do conteúdo nos currículos de graduação. De acordo com a literatura(16), a falta dessa abordagem pode influenciar em atitudes negativas frente aos fatores causadores do alcoolismo. Além disso, deve-se levar em consideração o fato de que o aluno pode refletir em suas atitudes o comportamento da sociedade e suas ações frente à problemática do alcoolismo ${ }^{(8)}$ e que, durante a graduação, deve haver a preocupação em diminuir a influência de ideias e preconceitos que o estudante possa trazer do seu meio social, pois, uma vez que ele traz consigo essas influências, o alcoolismo passa a ser visto por ele como algo exclusivamente moral $^{(17)}$. O mesmo acaba não levando em consideração os fatores biológicos que influenciam no alcoolismo, levando-os a julgamentos de valor que geram ou incentivam o aparecimento de posturas rígidas e punitivas em relação ao paciente.

Por fim, no que se refere ao fator 5 (bebida alcoólica), a alta proporção de respostas indiferente e discordo aos itens positivos, e a proporção praticamente igual de respostas aos itens positivos, pode nos levar a pensar em dois fatos: o primeiro é a tendência a atitudes negativas em relação a bebida alcoólica; o segundo, é, talvez, a falta de uma atitude segura dos estudantes de enfermagem em relação à bebida alcoólica. Ressalta-se que esse fator mede atitudes frente à bebida alcoólica; consequências trazidas pelo uso das bebidas ao individuo; o limite entre o beber e o normal e o patológico; e os efeitos da bebida sobre o comportamento da pessoa.

Com relação à tendência a atitudes negativas frente à bebida alcoólica, talvez esse resultado esteja relacionado às concepções e vivências proporcionadas por experiências desses estudantes de enfermagem ao prestarem assistência a esses pacientes com complicações decorrentes do beber durante estágios curriculares. Além disso, é possível que as informações recebidas durante a graduação em disciplinas de formação geral, que enfatizam o álcool como fator contribuinte e, muitas vezes, desencadeante de uma série de patologias orgânicas ${ }^{(8)}$ justifiquem tal achado.

A falta de uma atitude segura em relação aos itens negativos do fator 5 (bebida alcoólica) talvez se justifique, mais uma vez, pelo fato de que muitos estudantes de enfermagem refletem a visão dominante da sociedade, que aceita o beber moderado e rejeita o indivíduo que bebe de maneira descontrolada. Além disso, autores de um estudo realizado na Inglaterra ${ }^{(19)}$ demonstraram que a hesitação nas atitudes de enfermeiros em relação ao beber foi atribuída à aceitação difundida socialmente do beber moderado, bem como à comparação com o próprio consumo de álcool dos enfermeiros. Por isso, a falta de uma atitude definida pode estar relacionada ao próprio padrão de consumo do estudante de enfermagem, uma vez que pesquisa que observou o padrão de consumo por graduandos ${ }^{(20)}$ demonstrou que uma parcela considerável de estudantes da área de enfermagem apresentou níveis moderado e alto de consumo de bebidas alcoólicas.

Esse estudo possui limitações, como o envolvimento de somente duas faculdades de enfermagem, impossibilitando a generalização dos resultados; além do mais, poderia ter sido enriquecido com a investigação do padrão de ingestão de álcool dos participantes, possibilitando verificar a associação entre as atitudes apresentadas, principalmente com relação às atitudes frente às bebidas alcoólicas, e o padrão pessoal de uso de álcool.

No entanto, o estudo contribui para o conhecimento sobre a temática, principalmente frente à constatação de que investigações sobre esse tema são escassas no país, apesar dos avanços ocorridos no que se refere ao estudo das atitudes dos profissionais enfermeiros. Porém, quando o objeto são os estudantes de enfermagem, não se observa a mesma quantidade de pesquisas, o que causa uma lacuna na literatura da área.

Além das contribuições já citadas, essa pesquisa pode subsidiar a proposição de novos estudos que visem explorar mais as questões relacionas a atitudes de estudantes de enfermagem frente ao álcool, bem como ao ensino em enfermagem relacionado ao abuso de substâncias psicoativas.

\section{CONCLUSÃO}

Diante dos resultados apresentados no estudo em relação às atitudes dos estudantes de enfermagem frente à bebida alcoólica, ao alcoolismo e ao alcoolista, por meio de suas posições frente aos itens de uma escala de atitudes, observou-se que grande parte dos graduandos não apresentou atitude definida com relação à temática. No entanto, predominaram as tendências a atitudes negativas frente ao problema, uma vez que se observou, entre os participantes, uma predisposição em discordar dos itens positivos e em concordar com itens negativos da escala.

Uma vez que os estudantes de enfermagem apresentam atitudes negativas frente ao álcool, ao alcoolismo e ao alcoolista, tais atitudes podem se perpetuar após sua formação 
como enfermeiro, levando a um cuidado precário e carregado de preconceitos frente ao paciente dependente de álcool, presença constante e crescente nos serviços de saúde.

Conclui-se, ainda, que essas atitudes negativas devem-se, em grande parte, ao pouco preparo recebido durante a graduação. Nesse sentido, é premente a necessidade de que medidas sejam tomadas para que maior atenção seja dada à temática nos currículos de enfermagem.

\section{REFERÊNCIAS}

1. World Health Organization. The World Health Report 2002: Reducing Risks, Promoting Healthy Life. Geneva: World Health Organization; 2002.

2. Pan American Health Organization. (2007). Alcohol and Public Health in the Americas: a case for action. Washington, DC: Pan American Health Organization; 2007.

3. Carlini EA, Galduróz JCF, Noto AR, Nappo SA. I Levantamento domiciliar sobre uso de drogas psicotrópicas no Brasil. São Paulo: Centro Brasileiro de Informações sobre Drogas Psicotrópicas, Departamento de Psicobiologia da Universidade Federal de São Paulo. 2001.

4. Carlini EA, Galduróz JCF, Noto AR, Nappo SA. II Levantamento domiciliar sobre uso de drogas psicotrópicas no Brasil - São Paulo: Centro Brasileiro de Informações Sobre Drogas Psicotrópicas, Departamento de Psicobiologia da Universidade Federal de São Paulo. 2005.

5. Ministério da Saúde. A política do Ministério da Saúde de atenção integral a usuários de álcool e outras drogas. Brasília, DF: O Ministério; 2004.

6. Meloni JN, Laranjeira R. Custo social e de saúde do consumo de álcool. Rev Bras Psiquiatr 2004; 26Suppl1:7-10.

7. Noto AR, Moura YG, Nappo AS, Galduróz JCF, Carlini EA. Internações por transtornos mentais e de comportamento decorrentes de substâncias psicoativas: um estudo epidemiológico nacional do período de 1988 a 1999. J Bras Psiquiatr 2002 mar-abr;51(2):113-21.

8. Vargas D, Labate RC. Atitudes de enfermeiros de hospital geral frente ao uso do álcool e alcoolismo. Rev Bras Enferm 2006;59(1): 27-41.

9. Happell B, Taylor C. Drug and Alcohol Education for Nurses: Have We Examined the Whole Problem?. J Addictions Nurs 1999;11(4):180-185.

10. Sellek CS, Redding, B. Knowledge and Attitudes of registered nurses toward perinatal substance abuse. Journal of Obstetric, Gynecologic an Neonatal Nursing. 1998;27(1): 70-77.

11. Caetano R. Public opinions about alcoholism and its treatment. Journal of Studies on Alcohol. 1987; 48(2):153-160.

12. Edwards G. The Treatment of drinking Problems: a guide for the helping professions. 2 nd ed. Boston: Blackwell Scientific Publications; 1987.

13. Vilela MV, Ventura CAA, Silva EC. Conocimientos de estudiantes de enfermería sobre alcohol y drogas. Rev Latino-Am Enferm 2010;18(n. ${ }^{\circ}$ esp.):529-534.

14. Gil-Merlos AS. Aceitação e rejeição do alcoolismo: um estudo com alunos de enfermagem. Ribeirão Preto. Dissertação [Mestrado em Enfermgem] -Escola de Enfermagem de Ribeirão Preto-USP; 1985.
15. Assunção NA. Alcoolismo e ensino de enfermagem: convergências e divergências entre o discurso e a prática. Pelotas. Tese [Doutorado em Enfermagem] -Universidade Federal de Pelotas; 2000.

16. Pillon CS. O uso do álcool e a educação formal dos enfermeiros. São Paulo. 2003. Tese [Doutorado em Enfermagem] -Escola Paulista de Medicina-UNIFESP; 2003.

17. Luis MAV, Pillon SC, O conhecimento dos alunos de enfermagem sobre álcool e drogas. Rev Eletrônica Enferm 2003;5(1):21-27.

18. Miranda SP; Vargas D. Satisfação de pacientes de um centro de atenção Psicossocial álcool e drogas com o atendimento do enfermeiro. SMAD, Rev Eletrônica Saúde Mental Alcool Drog 2009;5(2):1-15.

19. Bae E. Lowery B. Patient and situtional factors that affect nursing studentes' like or deslike of caring for patients. Rev Nurs Res 1987;36(5):298-302.

20. Balan TG, Campos CJG. Padrão de consumo de bebidas alcoólicas entre graduandas de enfermagem de uma Universidade Estadual Paulista. SMAD, Rev. Eletrônica Saúde Mental Álcool Drog 2006;2(2).

\section{ERRATA}

No artigo "Álcool e alcoolismo: atitudes de estudantes de enfermagem", com número de DOI: 10.1590/S0034-71672013000100013, publicado no periódico Revista Brasileira de Enfermagem, v66(1):84-9, na página 89 onde se lia:

“18. Miranda SP, Vargas D. Satisfação de pacientes de um centro de atenção Psicossocial álcool e drogas com o atendimento do enfermeiro. SMAD, Rev Eletrônica Saúde Mental Alcool Drog 2019;5(2):1-15".

\section{Leia-se:}

“18. Miranda SP, Vargas D. Satisfação de pacientes de um centro de atenção Psicossocial álcool e drogas com o atendimento do enfermeiro. SMAD, Rev Eletrônica Saúde Mental Alcool Drog 2009;5(2):1-15.".

Rev Bras Enferm [Internet]. 2013 jan-fev;66(1):84-9. 